PharmacoEconomics \& Outcomes News 887, p19 - 18 Sep 2021

\title{
Oral HIV self-test kit distribution cost effective in South Africa?
}

Oral HIV self-test (HIVST) kit distribution under the Self-Testing AfRica (STAR) Initiative appears to be cost effective in various settings in South Africa, according to findings of two studies presented as abstracts at the 11th International AIDS Society Conference on HIV Science in July 2021.1,2

The first study evaluated the cost effectiveness of eleven HIVST distribution models in urban and rural settings over a period of 12 months (9 models) or less under the STAR Initiative between 2018 and 2019. Cost effectiveness was assessed from a South African provider perspective over a two-year time horizon. It was assumed that care cascaded from self-testing to initiation of antiretroviral (ART) therapy in positive cases, and over one million HIVST kits were distributed.

The self-test positivity rate ranged from $4 \%$ in a workplace model to $23 \%$ in a horizontal primary healthcare (PHC) model. The cost per HIVST kit ranged from \$4.87 in a sex worker model to \$18.07 in mobile integration model, and the cost per reactive HIVST was $\$ 28$ and $\$ 414$ in these models, respectively. The cost per confirmed case of HIV identified ranged from \$66 in the sex worker model to \$1229 in a vertical PHC model, and the cost per ART initiation was \$116 and $\$ 1278$ in these models, respectively.

"HIVST distribution cost varied widely across models, with the sex worker, transport hub, and workplace models being the most efficient and least costly distribution approaches," concluded the authors.

The second study evaluated the cost effectiveness of six HIVST distribution models (fixed-point, taxi ranks, workplace, partners of PHC index cases, partners of pregnant women, and primary PHC distribution) based on STAR Initiative data. Cost effectiveness was assessed from the provider perspective in South Africa over a 20-year time horizon, from 2020 to 2039, under two coverage scenarios: one million HIVST kits per year (status quo), or up to 6.3 million kits per year (target).

Secondary distribution to partners of PHC index cases had the greatest epidemiological impact but was one of the least cost-effective models, while workplace distribution was cost-saving (\$52-\$76 million) [2019 US dollars] but had only a moderate impact. It was estimated that scale-up of distribution to 6.3 million HIVST kits would lead to a threefold increase in life-years saved compared with the status quo.

"Optimisation-informed distributions have the potential to vastly improve the impact of HIVST. Using this approach, HIVST can play a key role in improving the long-term health impact of investment in HIVST, and assist in catching up testing targets post-COVID-19," said the authors.

1. Sande L, et al. The cost and intermediary cost-effectiveness of oral HIV self-test kit distribution across eleven distribution models in South Africa. 11th International AIDS Society Conference on HIV Science : abstr. OAC0501, 18 Jul 2021.

2. Jamieson L, et al. The cost effectiveness and optimal configuration of oral HIV self-test kit distribution in South Africa: a model analysis. 11th International AIDS Society Conference on HIV Science : abstr. PEC325, 18 Jul 2021. 